\title{
无线传感器网络传输控制协议
}

\author{
方维维，钱德沛，刘 轶
}

(北京航空航天大学 计算机学院 中德软件技术联合研究所,北京 100083)

\section{Transmission Control Protocols for Wireless Sensor Networks}

FANG Wei-Wei ${ }^{+}, \quad$ QIAN De-Pei, LIU Yi

(Sino-German Joint Software Institute, School of Computer Science, BeiHang University, Beijing 100083, China)

+ Corresponding author: E-mail: fangvv@gmail.com

Fang WW, Qian DP, Liu Y. Transmission control protocols for wireless sensor networks. Journal of Software, 2008,19(6):1439-1451. http://www.jos.org.cn/1000-9825/19/1439.htm

\begin{abstract}
This paper gives an introduction to the transmission control problem and a survey to the recent novel protocols and their taxonomy. Firstly, the research background of the WSN transmission control problem is presented. Then a comprehensive survey with analysis is made to different methods and representative protocols in two aspects: Congestion control and reliability guarantee, respectively. In the end, the protocols are compared and future research directions are suggested.
\end{abstract}

Key words: wireless sensor networks; congestion control; reliability guarantee; acknowledgement mechanism; QoS

摘 要: 介绍了无线传感器网络的传输控制问题,总结了近年来提出的各种传输控制协议及主要设计思想.首先 引入了传输控制问题的研究背景; 然后分别从拥塞控制和可靠保证两个方面介绍代表性的研究工作, 对涉及的主要 方法进行分类分析;最后进行了各种协议的综合比较,并指出了无线传感器网络传输控制未来的研究方向.

关键词: 无线传感器网络;拥塞控制;可靠保证;反馈机制;服务质量

中图法分类号: TP393 文献标识码: A

无线传感器网络(wireless sensor networks, 简称 WSNs) 由大量具备数据处理和通信能力的传感器节点组成, 其目的是协作的感知、采集网络覆盖范围内监测对象的相关信息,并通过短距离无线多跳的通信方式将监测数 据发送给网关,提供给用户进行分析和处理 ${ }^{[1]}$.同时,用户也可以根据网络应用需求,通过网关向传感器节点发送 控制数据包,完成信息查询和网络管理维护等任务.在传感器节点能量储备和计算能力等资源普遍受限的条件 下,能否为网络中的数据传输提供可靠的传输控制机制, 以保证信息的有效获取和网络的优化管理,就成为 WSN 中一个基本问题,也是衡量 WSN 网络服务质量(QoS)的一个重要指标 ${ }^{[2]}$.

近年来,国内外的研究机构已经开展了 WSN 传输控制方面的研究工作,取得了一定的研究进展.本文综述

* Supported by the National Natural Science Foundation of China under Grant Nos.90412011, 90612004, 60673180 (国家自然科学基 金); the International Science and Technology Cooperative Program of China under Grant No.2006DFA11080 (科技部国际科技合作计划 项目); the Research Program of Federal Ministry of Education and Research of Germany under Grant No.01BU0680 (德国教研部资助 项目)

Received 2007-07-22; Accepted 2007-12-24 
近年来在这一领域取得的研究成果. 文章首先引入相关的研究背景, 在详细综述和比较已有的 WSN 传输控制协 议和方案的基础上,指出未来的研究方向.

\section{1 无线传感器网络传输控制协议的研究背景}

\section{1 应用传输控制的原因}

在 WSN 中,存在着如下影响数据传输的负面因素 ${ }^{[3,4]}$ :

(1) WSN 的无线链路是开放的有损传播介质, 存在着多径衰落和阴影效应(由于通信范围有限, 路径损耗 较低,一般可忽略不计),加之其信道一般采用开放的 ISM 频段,使得网络传输的误码率较高;

(2) 同一区域中的多个传感器节点之间同时进行通信, 节点在接收数据时易受到其他传输信号的干扰;

(3) 由于能量耗尽、节点移动或遭到外来破坏等原因,造成传感器节点死亡和传输路径失效;

（4）传感器节点的存储资源极其有限, 在网络流量过大时,容易导致协议栈内的数据包存储缓冲区溢出.

因此,WSN 必须提供一定的传输控制机制, 以保证网络传输效率.这些机制主要可分为拥塞控制(congestion control)和可靠保证(reliability guarantee)两大类 ${ }^{[3]}$. 拥塞控制用于把网络从拥塞状态中恢复出来,使负载不超过 网络的传送能力 ${ }^{[5]}$; 可靠保证用于解决数据包传输丢失的问题,使接收端可以获取完整而有效的数据信息.

\section{2 传统协议的不足之处}

传统 IP 网络主要使用协议栈中传输层的 UDP 和 TCP 协议控制数据传输.UDP 协议是面向无连接的传输 协议, 不提供对数据包的流量控制及错误恢复; TCP 协议则提供了可靠的传输保证, 如利用滑动窗口和 AIMD 等 机制进行拥塞控制, 以及使用重传进行差错控制 ${ }^{[6]}$.但 TCP 协议却不能直接用于 WSN, 主要原因如下 ${ }^{[3,7,8]}$ :

(1) TCP 协议遵循端到端(end-to-end)的设计思想,数据包的传输控制任务被赋于网络的端节点上,中间节点 只承担数据包的转发. 而 WSN 以数据为中心, 中间节点可能会对相关数据进行在网处理(in-network processing), 即根据数据相关性对多个数据包内的信息进行综合处理,得到新的数据包发送给接收端,直接使用 TCP 协议会 导致将此视为丢包而引发重传.

(2) TCP 协议建立和释放连接的握手机制相对比较复杂, 耗时较长, 不利于传感器节点及时反馈被监测对象 的相关信息.WSN 网络拓扑的动态变化也给 TCP 连接状态的建立和维护带来了一定的困难.

（3） TCP 协议采用基于数据包(packet-based)的可靠性度量,即尽力保证所有发出的数据包都被接收节点正 确收到.在 WSN 中,可能会有多个传感器节点监测同一对象,使得监测数据具有很强的午余性和关联性. 只要最 终获取的监测信息能够描述对象的真实状况,具有一定的逼真度(fidelity)就可以,并不一定要求数据包传输的 完全可靠 ${ }^{[9,10]}$,这种方式也被称为基于事件(event-based)的可靠性度量 ${ }^{[9]}$.

(4) TCP 协议中数据包重传通过端节点之间的 ACK 反馈和超时机制来保证. 传感器网络数据包中所含的数 据量相对较小, 大量 ACK 包的传输会加重传输负载和能量消耗. 并且, 每次 ACK 确认和数据包重传都要从发送 端发出经历多跳传输路径到达目的端,引发整条路径上所有节点的能量消耗.

(5) WSN 中非拥塞丢包和多路传输等引起的数据包传输乱序, 都会引发 TCP 协议的错误响应, 使得发送端 频频进入拥塞控制阶段,导致传输性能下降.

(6) TCP 协议要求每个网络节点具有独一无二或全网独立的网络地址. 在大规模的 WSN 中, 为了减少长地 址位带来的传输消耗,传感器节点可能只具有局部独立的或地理位置相关的网络地址 ${ }^{[11]}$ 或采用无网络地址的 传输方案 ${ }^{[12]}$,无法直接使用 TCP 协议.

WSN 的应用需求对传输控制协议的设计提出了很高的要求,但目前学术界对于 WSN 技术的研究尚处于 起步和发展阶段,协议体系并不完整.尽管出现了少量综合处理网络拥塞和提供可靠保证的协议 ${ }^{[13,14]}$,但它们主 要是基于 TCP 协议进行的部分改进.当前的研究工作仍主要侧重于拥塞控制或可靠保证两者之一.基于本文的 主要内容,图 1 进行了 WSN 传输控制问题各种相关协议和方案的分类总结. 


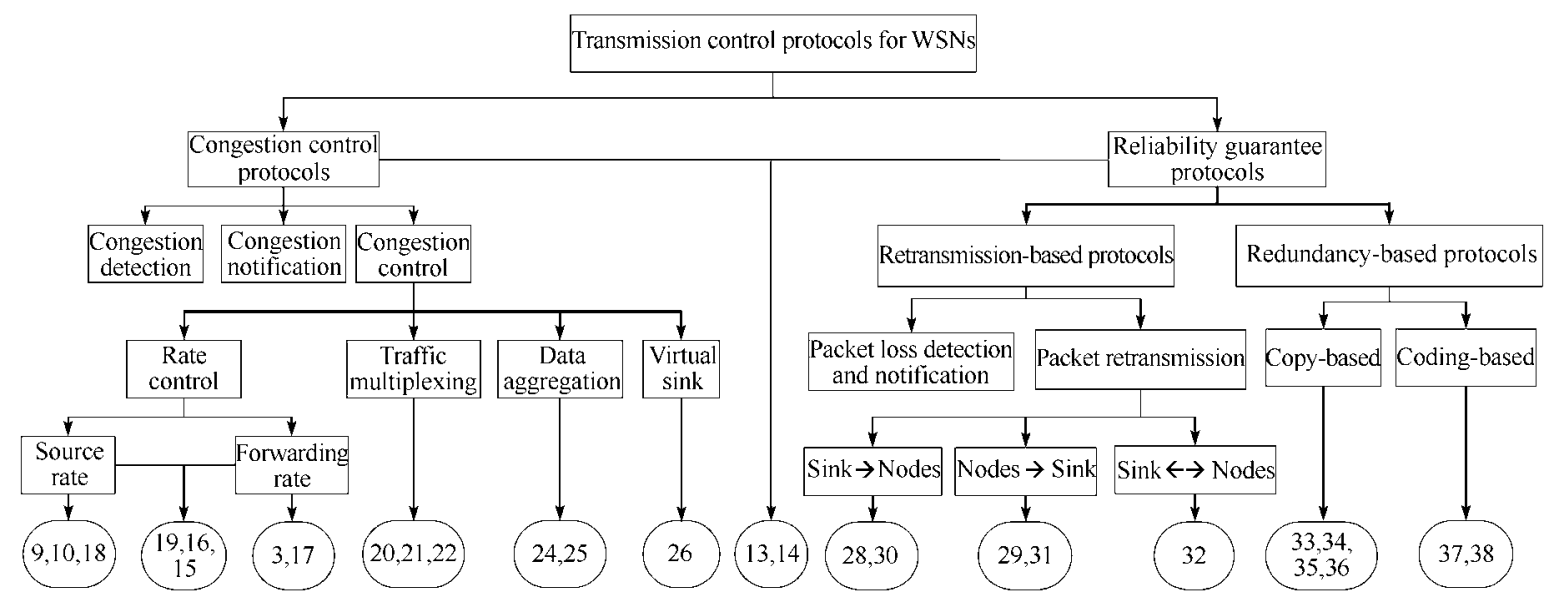

Fig.1 WSN transmission protocols: A taxonomy

图 1 无线传感器网络传输控制协议分类

\section{2 无线传感器网络拥塞控制协议}

\section{1 拥塞检测}

准确、高效的拥塞检测是进行拥塞控制的前提和基础.目前,WSN 中主要采用如下方法进行拥塞检测:

(1) 缓冲区占用 ${ }^{[9]}$ : 根据节点内部数据包存储缓冲区的占用情况来检测拥塞是否发生. 对缓冲区大小为 $B$ 的节点, 定义 $b_{k}$ 为第 $k$ 个时间间隔结束时其缓冲区的占用大小, 若 $\left[b_{k}+\left(b_{k}-b_{k-1}\right)\right]>B$, 则推测在第 $(k+1)$ 个 间隔内将发生拥塞.这也是传统网络中网络节点进行拥塞检测的主要方法.然而,由于缺乏对无线信道 繁忙程度的估计,在 WSN 中该方法不能很准确地预测拥塞 ${ }^{[13]}$.

(2) 信道负载 ${ }^{[15]}$ : 节点通过监听信道是否空闲来判断自身是否处于拥塞状态. 该方法准确程度较高, 缺点在 于长时间监听信道会加大节点的能量开销.

(3) 缓冲区和信道 ${ }^{[15]}$ :为了克服方法(1)、(2)各自的缺点,文献[15]提出了将两者结合的方法,即在缓冲区非 空时进行信道状态周期性采样,在准确检测拥塞的同时减轻了能量开销.

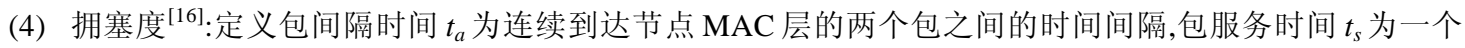
包从到达 MAC 层到其被成功发送的时间间隔,拥塞度为平均包服务时间和平均包间隔时间的比率, 即对节点 $i, d(i)=t_{s} / t_{a} . d(i)$ 的值是否大于 1 反映了节点 $i$ 是否处于发生拥塞的趋势中.

若节点发现自身处于拥塞状态,并且需要将此信息传播给邻居节点或上游节点来进行某些控制操作, 一般 采用如下两种方式来报告拥塞消息 ${ }^{[16]}$ :

(1) 明文方式(explicit congestion notification,简称 ECN): 节点发送包含拥塞消息的特定类型的控制包 ${ }^{[15]}$. 为了加快该消息的扩散速度,可以通过设定 MAC 层竞争参数来增大其访问信道的优先权 ${ }^{[17]}$. 缺点是 控制包带来了额外的传输开销.

(2) 捎带方式(implicit congestion notification,简称 ICN):利用无线信道的广播特性,将拥塞状态信息捎带在 正要传输的数据包包头中, 邻居节点通过监听(overhear)通信范围内的数据传输获取相关信息 ${ }^{[16]}$.与明 文方式相比,捎带方式减轻了网络负载,但增加了监听数据传输和处理数据包的开销.

\section{2 控制协议}

当传感器节点检测到拥塞发生后,将会综合采用各种控制机制减轻拥塞带来的负面影响,提高数据传输效 率.我们选择了一些典型的控制协议,按照其采用的核心控制机制进行分类介绍和分析. 


\subsection{1 流量控制}

(1) 报告速率调节

一般来说,WSN 的播撒密度较高,数据具有很强的关联性和午余度.但用户一般只关心网络整体返回的监 测信息的准确度,而非单个节点的报告. 因此, 只要保证获取的信息足够描述被监测对象的状态,具有一定的逼 真度,就可以对相关数据源节点的报告频率进行调整, 以便在发生拥塞时减轻网络的流量压力.

ESRT 协议 ${ }^{[9]}$ 是第一个基于速率调节的拥塞控制协议.该协议定义 $f$ 为源节点的报告速率, 可靠性度量 $\eta=r_{i} / R$, 其中 $r_{i}$ 为在第 $i$ 个时间间隔网关收到的包数量, $R$ 为达到可靠监测所需要收到的包数量. 当 $\eta>1$ 时,网络达 到高可靠状态(high reliability, 简称 HR), 反之则为低可靠状态(low reliability,简称 LR). 同时,定义第 $i$ 个时间间隔 时的网络状态(按照报告速率 $f$ 递增引发的状态变化进行排序):

$$
S_{i} \in\{(N C, L R),(N C, H R), O O R,(C, H R),(C, L R)\} .
$$

其中, $N=$ No, $C=$ Congestion, $L=$ low $, H=h i g h, R=$ Reliability, $O O R=$ Optimal Operating Region. 随着报告速率 $f$ 的变化, $S_{i}$ 可以向邻近的其他状态转化. 协议参考 $S_{i}$ 来调整第 $(i+1)$ 个时间间隔的报告速率 $f_{i+1}$, 调整算法如下:

$$
f_{i+1}=\left\{\begin{array}{ll}
\frac{f_{i}}{\eta_{i}}, & S_{i}=(N C, L R) \\
\frac{f_{i}}{2}\left(1+\frac{1}{\eta_{i}}\right), & S_{i}=(N C, H R) \\
f_{i}, & S_{i}=(O O R) \\
\frac{f_{i}}{\eta_{i}}, & S_{i}=(C, H R) \\
f_{i}^{\left(\eta^{i} / k\right)}, & S_{i}=(C, L R)
\end{array} .\right.
$$

ESRT 综合考虑了网络对于可靠性和能耗的要求,通过对报告速率的适当调整,达到了在减轻拥塞的同时 提高可靠性和节省能量的目的.经过连续多个时间间隔的调整和网络状态转化,使网络尽量维持在 OOR 状态. 新的调整设置方案将由网关通过广播的方式通知给当前所有的数据源节点进行设置.ESRT 的缺点在于需要网 关具有覆盖整个网络的通信能力,使用简单的包数量统计的方式来衡量可靠性,且不区分数据源对信息逼真度 的贡献能力,对所有节点采用统一的调整方案.

针对 ESRT 协议的缺点,PORT 协议 ${ }^{[10]}$ 将报告速率调整问题建模为优化问题.网关根据各数据源节点对信息 逼真度的贡献情况对当前的 $m$ 个数据源节点进行速率调整, 在保证逼真度的前提下, 使网络总消耗 $\sum_{1}^{m}\left(t_{i} \times p_{i}\right)$ 达 到最小,其中 $t_{i}$ 为节点 $i$ 的发送速率, $p_{i}$ 为通信消耗. 调整方案通过多跳传输或网关广播发送给各个数据源节点.

IFRC 协议 ${ }^{[18]}$ 采用 AIMD 机制来进行报告速率控制. 定义源节点 $i$ 的报告速率 $r_{i}$, 辅助参数 $\delta$. 若无拥塞发生, 则每经过 $1 / r_{i}$ 秒,则 $r_{i}=r_{i}+\delta / r_{i}$; 一旦拥塞发生,则 $r_{i}=r_{i} / 2$. 通过在包头中包含一些拥塞相关的标识字段, IFRC 实现 了和周围邻居节点之间的拥塞信息共享,并基于此定义了两条规则来保证节点间速率调整的公平性: (1) 节点 $i$ 的报告速率不能超过父节点 $j$; (2) 当 $i$ 的一个邻居 $j$ 发生了拥塞时, $i$ 将自身的报告速率设置为 $r_{i}$ 和 $r_{j}$ 中的较小 值. 同理, 若 $i$ 的多个子节点发生了拥塞,则 $i$ 将自身的报告速率设置为 $r_{i}$ 和 $r_{l}$ 中的较小值, 其中 $l$ 是拥塞程度最严 重的子节点.IFRC 协议主要侧重于防止 AIMD 调节方式带来的不公平性 ${ }^{[3]}$, 使得相邻的多个节点能够更公平地 分享无线信道带宽进行数据传输.

(2) 转发速率调节

若网络对数据采集的逼真度要求较高,则一般不适用于报告速率调节,而是选择在流量汇聚而发生拥塞的

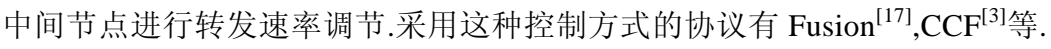

Fusion 协议 ${ }^{[17]}$ 采用令牌桶(token bucket)的方式进行流量整形,节点在监听到父节点转发了 $N$ 个数据包后 积累一个令牌, 在令牌数不为 0 时才允许向父节点发送数据, 每次数据发送将会消耗一个令牌.CCF 协议 ${ }^{[3]}$ 提出 了速率比较的方法, 节点测量自身可达的平均发送速率 $r$, 其子孙节点的最高允许速率为 $r_{d a t a}=r / n(n$ 为自身和所 
有子孙节点数目的总和), 在拥塞发生时,节点选择自身产生的 $r_{\text {data }}$ 和父节点告知的 $r_{\text {data,parent }}$ 中较小的值, 并发送 给子节点供其调整. 这两种协议主要依靠多到一的树状传输结构中父子节点之间的协作能力,需要节点在传输 时保持彼此间稳定的父子关系.

然而,仅依靠调节转发速率将会导致拥塞状态沿着数据传输的相反方向不断传递,最终到达数据源节点. 若 数据源节点不能支持报告速率调节,将会导致丢包现象的发生.

(3) 综合速率调节

在多跳结构的 WSN 中,传感器节点承担着数据采集和路由转发的双重任务. 当拥塞发生时,仅通过单一的 速率调节方式往往不能达到有效的控制效果,或不能尽量保证监测信息的逼真度.因此,研究人员提出了 Buffer-based ${ }^{[19]}$, PCCP $^{[16]}$ 和 CODA $^{[15]}$ 等综合调节两种速率的控制协议.

Buffer-based 协议 ${ }^{[19]}$ 采用了一种基于缓冲区管理的轻量级控制机制. 节点在传输数据包时将当前自身的数 据包缓冲区占用情况捎带在包头中发送,所有邻居节点通过监听数据包传输获得相关信息.在向下游邻居节点 发送数据之前,节点都要检查该邻居节点的缓冲区是否已经溢出,若是,则暂停数据发送,直到监听到该邻居节 点缓冲不为满状态时,再继续发送.拥塞状态会不断向数据源传递,最终通过调节数据源节点的报告速率减轻拥 塞. 该协议还结合了多路转发路由和设置多网关的方法,增加拥塞时备选的下游节点数目, 提高网络的传输 效率.

PCCP 协议 ${ }^{[16]}$ 按照数据源的重要性(如感知数据类型等)对流进行分级, 采用加权优先的方式保证调整公平 性. 协议设置了两个队列来分别缓存报告和转发的数据包,使用 WFQ 或 WRR 算法来保证对两个队列中包的公 平调度.对每个节点 $i$, 定义其报告速率 $r_{s r c}^{i}$ 和调度速率 $r_{s v c}^{i}$, 同时定义 3 种优先级:源优先级 $S P(i)$ 为其自身产生 的流的优先级,转发流优先级 $T P(i)$ 为所有 $i$ 的下游节点转发给 $i$ 的数据流优先级之和,全局优先级 $G P(i)=S P(i)+T P(i)$.协议运行时,节点 $i$ 的父节点 $P_{i, 0}$ 将通过收发速率比的方法得到的拥塞度信息 $d\left(P_{i, 0}\right)$ 通过包头 捎带的方式告知 $i$, 子节点 $i$ 应用如下算法进行调整 $\left(O\left(P_{i, 0}\right)\right.$ 为节点 $P_{i, 0}$ 下游当前的数据源节点数目):

$$
\begin{aligned}
& r_{r v c}^{i}=\left\{\begin{array}{ll}
\min \left(\frac{r_{r v}^{i}}{d\left(P_{i, 0}\right.}, \frac{1}{t_{s}^{i}}\right), & O\left(P_{i, 0}\right) \text { 减小或 } O\left(P_{i, 0}\right) \text { 不变, } d\left(P_{i, 0}\right) \text { 减小 } \\
\min \left(\frac{1}{t_{s}^{P_{i, 0}}} \times \frac{G P(i)}{G P\left(P_{i, 0}\right)}, \frac{1}{t_{s}^{i}}\right), & O\left(P_{i, 0}\right) \text { 增大或 } O\left(P_{i, 0}\right) \text { 不变, } d\left(P_{i, 0}\right) \text { 增大 }
\end{array},\right. \\
& r_{\text {src }}^{i}=r_{r v c}^{i} \times \frac{T P(i)}{G P(i)} .
\end{aligned}
$$

通过报告速率的调节,PCCP 协议保证具有相同的源优先级的节点可以获得相同的报告速率, 而源优先级 高的节点可以获得更高的速率和带宽.但是,它要求在数据包中增加一定长度的优先级标识位,带来了额外的传 输开销.另外,它仅从数据流的加权公平性角度调整报告速率,并不能很可靠地保证监测信息的逼真度.

CODA 协议 ${ }^{[15]}$ 结合了开环控制和闭环控制这两种方式来解决拥塞问题.在由突发的网络流量交织导致发 生局部短暂性拥塞时,将启用开环控制策略.检测到拥塞发生的节点沿着向数据源节点的方向向上游节点扩散 后压消息(suppression message),收到此消息的节点将根据本地的网络状况判断是否继续向其上游节点传播,同 时,采取一定的本地控制策略,如丢弃部分数据包,降低报告或转发速率,路由改道等来减轻拥塞.同时,若某个被 监测事件的发生频率低于信道吞吐量的设定阈值(比例),则源节点可以自行调节报告速率,否则便触发闭环拥 塞控制机制.此时,负责监测此事件的节点在发送的消息包头中设置调整位,网关收到这样的包之后,将会对监 测该事件的所有相关源节点的报告速率进行综合调整,将调整方案以 ACK 包的形式反馈给各相关节点. 与 ESRT 协议相比,CODA 协议的报告速率调节只是在节点发现自身监测的事件会引起拥塞而主动请求网关进行 协调时才起作用, 且只针对监测该事件的所有节点作调整. 相对而言, 开环控制策略用以快速减轻多数据流交汇 而引发的局部短暂拥塞,而闭环控制机制适用于调整因数据率太高使得网络无法承载而发生长时间拥塞的情 况,但由于涉及网关和多个节点的反馈交互,在大规模网络中闭环控制调整速度相对较慢. 


\subsection{2 多路分流}

由于传感器网络节点的资源受限, 现有的路由协议大多采用单路径单播数据转发机制, 即根据能量消耗、 路径跳数等衡量指标选择一条相对最优路径进行数据发送.这样的路由方式导致了网络流量集中在该条最优 路径上,容易在多条传输路径交汇时引发拥塞现象. 若交汇的数据流分别流向不同的目的节点或网关,可以采用 多路径转发的方式分散流量以解决此类拥塞问题 ${ }^{[20-22]}$.

ARC 协议 ${ }^{[20]}$ 利用传感器网络中存在的午余节点来构建新的转发路径.网络中的冗余节点利用休眠机制来 节省能量,但它们保持周期性的醒来,通过监听通信范围内的数据传输, 获取并保存数据包头中捎带的邻居节点 的拥塞程度、剩余能量和转发的流编号等信息.同时, 兄余节点将根据其邻居节点的拥塞程度大小来决定休眠 周期长短直至完全处于工作状态,这样就相当于在拥塞区域周围预置了多条可用的转发路径.拥塞发生时,拥塞 信息将沿着原数据传输路径的相反方向向上游节点反馈,寻找符合条件的节点进行分流.从分流节点分散出的 数据包流将会沿着拥塞区域边界向目的节点的方向进行传输,绕开拥塞区域后逐渐向原传输路径上的节点靠 拢,直至重新汇聚后发送到网关.流量汇聚节点通过数据包包头捎带的相关信息判断拥塞解除之后,将向数据分 流点发送解除多路分流状态的通知消息, 重新使用原来的单条最优路径进行传输, 以保证传输效率并节省传输 能量.

CAR 协议 ${ }^{[21]}$ 提出了与 ARC 协议相近的方法,较适用于存在多种不同传输优先级的数据流的网络中.协议 在发生拥塞时只在拥塞区域内转发优先级较高的数据流,而将低优先级的数据流分散到非拥塞区域中建立的 长路径上进行转发,这样就为不同的数据提供了区分式服务保证.

BGR 协议 ${ }^{[2]}$ 采用了在地理路由中增加方向偏离范围的方法来扩大转发路径的选择范围,节点随机选择此 范围内的邻居节点转发数据. 与其他协议相比, 其实现机制较为简单, 但随机选择的方式缺乏对邻居节点当前状 况(如能耗等)的综合考虑.另外,也存在将部分流量转发到周围其他拥塞区域的可能性.

多路分流的方法避免了流量控制可能带来的监测数据逼真度下降的问题,但分流路径往往不是符合路由 选择标准的最优效率路径. 该类方法不适用于只有单个网关的 WSN 场景.

\subsection{3 数据聚合}

为了减少网络中传输的数据量, 延长网络寿命, 传感器节点可以使用数据聚合(data aggregation)技术 ${ }^{[23]}$, 对 多个内容存在关联的数据包进行综合处理, 组合出更有效或更符合用户需求的数据包再继续传输. 这种技术也 可以作为一种有效的控制方法来减轻网络拥塞 ${ }^{[24,25]}$.

CONCERT 协议 ${ }^{[24]}$ 采用适应性的数据聚合,减少网络中传输的数据量以减轻拥塞. 由于数据聚合节点的引 入会增加数据处理的时间开销,CONCERT 协议只在事先预测可能发生拥塞的区域布置聚合节点. 若无法准确 获知此信息,则聚合节点将通过移动来动态部署. 数据聚合节点将根据数据包中所含的时间、地域等关联性信 息进行聚合操作,同时,尽力保证监测数据的逼真度.

在 PREI 协议 ${ }^{[25]}$ 中, 整个网络覆盖区域被均匀地划分为多个大小相同的网格,聚合节点将对来自于同一个 网格的监测数据进行检查,剔除异常数据之后再对剩下的数据进行聚合操作.PREI 协议还引入了多级聚合的思 想, 对相邻网格的数据进行再次聚合, 进一步减少传输的数据量, 以保证监测数据量突增情况下的传输效率.

\section{2 .4 虚拟网关}

WSN 一般采用多对一的通信结构,因而靠近网关的节点承载的网络流量较大,容易引发拥塞,这一现象被 称为 “漏斗效应” ”26]. 为了解决这一问题,Siphon 协议 ${ }^{[26]}$ 提出在网络中布置少量具有双通信模块的传感器节点作 为虚拟网关,在发生拥塞时启动长距离通信模块(如 IEEE 802.11 网卡)和原网关进行通信,对网络流量进行分流. 协议操作分为 4 步: 1) 原网关初始化虚拟网关发现过程,虚拟网关在邻近范围内广播自身存在信息,传感器节 点收到信息后将建立到达虚拟网关的路由;(2) 拥塞检测; (3) 拥塞发生后,传感器节点将流量转发给附近的虚拟 网关,再通过虚拟网关之间的配合操作转发给原网关; (4) 若虚拟网关之间的长距离通信网络也发生了拥塞,那 么将采取一定的 MAC 层调节机制或缩小初始广播范围来进行控制.作者实现了基于 Stargate 虚拟网关 ${ }^{[27]}$ 和 Mica2 传感器节点 ${ }^{[27]}$ 的原型系统.Siphon 协议的缺点是需要增加额外的通信硬件, 虚拟网关的有效部署(如数量, 
位置,通信范围等)也是一个值得关注的问题.

\section{3 无线传感器网络可靠保证协议}

WSN 中的可靠保证协议主要分为两类:在基于数据重传的协议中,节点需要暂时缓存已发送的数据包, 并 使用重传控制机制来重传网络传输过程中丢失的数据包;基于冗余发送的协议主要采用信息冗余的方式保证 可靠性,节点无须缓存数据包和等待重传,但网络负载和能量开销相对较大.

\section{1 数据重传}

\subsection{1 丢包检测}

传感器节点主要通过接收到的数据包包头中相关序列号字段的连续性进行丢包检测,发现数据包丢失后 将信息反馈给当前持有该数据包的发送节点请求重传.丢包信息的反馈方式主要有以下 3 种 ${ }^{[2]}$ :

(1) ACK (acknowledge)方式:源节点为发送的每一数据包设置缓存和相应的重发定时器. 若在定时器超时之 前收到来自目的节点对此数据包的 ACK 控制包,则认为此数据包已经成功地传送,此时, 取消对该数据包的缓 存和定时; 否则, 将重发此数据包并重新设置定时器. 对于每个数据包, 接收节点都需要反馈 ACK, 负载和能耗 较大.

(2) NACK(negative ACK)方式:源节点缓存发送的数据包,但无须设置定时器. 若目的节点正确收到数据包, 则不反馈任何确认指示; 若目的节点通过检测数据包序列号检测到数据包的丢失, 则反馈 NACK 控制包, 要求重 发相应的数据包.NACK 只需针对少量丢失的数据包反馈, 减轻了 ACK 方式的负载和消耗.缺点在于,目的节点 必须知道每次传输的界限,即首包和末包的序列号,使其不能保证单包发送时的可靠性.

(3) IACK(implicit ACK)方式:发送节点缓存数据包,监听接收节点的数据传输, 若发现接收节点发送出该数 据包给其下一跳节点,则取消缓存. 这种方式不需要传输控制包,负载和消耗最小,但只能在单跳以内使用,且需 要节点能够正确地监听到邻居节点的传输情况,不适用于 TDMA 类 MAC 协议.

\subsection{2 重传协议}

网络中的数据重传方式主要有两种:端到端(end-to-end)重传和逐跳(hop-by-hop)重传.基于端到端控制方式 的典型协议,如 TCP,STCP ${ }^{[13]}$ 等,主要依靠目的端节点检测丢包,将丢包信息反馈给数据源节点进行重传处理.控 制包和重传数据包的传输需要经历整条传输路径, 不但降低了数据重传的可靠性和效率, 也加大了网络负载和 能量消耗 ${ }^{[28,29]}$. 同时,基于端节点的控制方式使得反馈处理时间相对较长,不利于数据的实时传输,节点难以对 传输状态,如 RTT 等进行有效的维护. 因此, 在 WSN 中较多地采用了逐跳控制方法,即在每跳传输的过程中,相邻 转发节点之间进行丢包检测和重传操作.下面根据数据流的发送方向,介绍几种典型的数据重传协议.

(1) 网关向节点

在 WSN 中,用户一般使用广播或多播的方式,通过网关向整个网络或局部网络内的多个节点发送查询、重 编程等控制消息.如果一条控制消息的数据量较大,则消息将被分片并使用多个数据包进行传输.

PSFQ 协议 ${ }^{[28]}$ 采用缓发快取 (pump slowly,fetch quickly)的方式进行传输控制. 在该协议中,传感器节点采用 广播的方式向邻居节点转发从网关发送出的控制数据包.缓发是指节点在向邻居节点广播数据包时,设定连续 广播的时间间隔为 $T \in\left(T_{\min }, T_{\max }\right)$, 这样的设置保证了数据包能够有一定的缓存时间以备重传,同时,节点可以通 过监听邻居节点数据传输减少数据的广播冗余; 快取是指节点发现丢包后暂停数据转发,将多个丢失包的序列 号信息综合在一个 NACK 控制包中广播给邻居节点.若重传后没有收到所有丢失的包,则按较短的时间周期 $T_{r}\left(T_{r}<T_{\max }\right)$ 继续广播多次,若仍失败,则将该请求向网关方向的节点传播,直到从这些节点收到所有丢失的包.在 某些情况下, 高序列号的包可能会完全丢失, 为了解决 NACK 传输界限的问题,保证数据的完整性,PSFQ 增加了 主动取包的功能:节点根据数据包头中捎带的该次传输数据包总量信息,在等待 $T_{\text {pro }}$ 时间后主动发送 NACK 包 请求丢失的数据块.在该协议中,传感器节点需要维护的计时器数量较多,重传控制过程较为复杂.

GARUDA 协议 ${ }^{[30]}$ 建立层次型网络结构,进行阶段性的丢包恢复操作.在网络形成的初始阶段,网关向网络 广播包含 bId 的控制包 (bId 初始值由网关设定),建立以核心节点 (bId 值为 3 的倍数的节点)为中心的层次结构. 
网关发送出的每条控制消息将分由多个数据包传输.核心节点在转发某条消息的数据包时,将在包头中包含 A-map 字段,用位标识的方法来表明自身已经收到了哪些属于该消息的数据包.丢包恢复的过程分为两个阶段: 下游的核心节点在收到上游的核心节点转发的数据包之后,检查 A-map 字段,发现有自身需要的丢失数据包则 向该上游节点发送 NACK 包请求重传; 非核心节点监听自身依附的核心节点的数据传输,在监听到属于某条消 息的所有数据包都被完整接收后(整个A-map 字段都被标识)再向核心节点发送重传请求.由于核心节点相对负 载较大,因此协议规定, 网关可以在更新层次结构时选择不同的初始 bId 值, 以保证网络的负载均衡和公平性.在 协议建立的层次结构中,核心节点数量较少,其丢包恢复的处理速度较快,保证了消息的及时获取; 非核心节点 可以从邻近的核心节点较为可靠地获取完整的消息,减轻了整个网络内重传竞争的压力.同时,根据 A-map 字段 标识位来判断是否请求重传的方式避免了 NACK 内爆问题 ${ }^{[19,20]}$ (在上游某个节点处发生丢包引发下游传输路 径上的所有节点接连发送 NACK 请求重传的现象)的发生.但 GARUDA 协议解决 NACK 传输界限问题要求传 感器节点必须安装能够收发周期性脉冲的设备, 用来在发送数据前进行预先交互, 增加了节点的设计复杂度. 另 外,初始阶段的 bId 包的广播以及数据包头中加入的 A-map 字段也加重了网络的传输负载和能耗.

(2) 节点向网关

RMST 协议 ${ }^{[29]}$ 是结合单播路由协议设计的可靠保证协议. 协议改进了原路由中只有从数据源到网关的单 向路径的方式,增加了用于反馈丢包信息的后向路径(back channel).传输层建立 NACK 机制反馈丢包信息,支持 节点缓存数据包和不缓存数据包两种操作方式:缓存方式下将采用逐跳方式进行重传,不缓存方式下采用端到 端的方式进行重传. 另外,协议还规定了 MAC 层提供选择性重传响应的方式(ARQ), 使用 ACK 对单播数据包进 行确认, 以协助传输层进一步提高传输的可靠性.

$\mathrm{RBC}$ 协议 ${ }^{[31]}$ 使用渐缩窗口的发送虚拟队列控制数据包的发送和重传.虚拟队列共分 $(M+2)$ 级,队列 $Q_{0} \sim Q_{m}$ 用来存储等待发送的数据包, 每个数据包占用队列中的一个存储单元, $Q_{m+1}$ 用来存放未占用的存储单元. 数据包 进入缓冲区之后,首先从 $Q_{m+1}$ 中找到可用的存储单元,然后移动到 $Q_{0}$ 的尾部等待发送,被发送之后移动到当前 队列的下一队列尾部, 若发送成功,则释放占用的存储单元到 $Q_{m+1}$, 否则, 将在此位置等待重发. 节点每次挑选当 前级别最低的非空队列首部的数据包进行发送.RBC 协议使用 IACK 的方式进行丢包控制,数据包发送时将捎 带自身和邻近的存储单元的 id 号, 接收节点通过检查发送节点连续发送的数据包中 id 号的连续性来检测丢包. 同时,接收节点在转发时捎带自身已经收到的数据包的存储单元 id 号,发送节点通过监听获取这些确认信息. 由 于网关无法通过 IACK 的方式反馈信息,则采用主动的 ACK 方式向邻居节点广播一段时间内收到的所有数据 包的确认.RBC 协议解决了滑动窗口方式中因为等待数据确认和重传包而引起的后续数据包持续长时间等待 发送的问题, 提高了发送效率. 其缺点是队列结构比较复杂, 节点进行数据传输监听和数据包的处理开销较大.

(3) 双向可靠保证

BRTM 协议 ${ }^{[32]}$ 针对不同数据发送方向上数据流对可靠性的不同要求, 提出了双向可靠保证协议.网关发送 给节点的查询包对可靠性要求较高,网关在包头加入特定标识位以表明传输界限, 节点采用 NACK 方式向网关 反馈丢包信息; 节点到网关的监测数据包几余性较高,可靠性要求相对较低,网关使用选择性 ACK 的方式进行 控制, 即只对数据值变化较大的部分数据包进行确认. 其缺点在于使用了端到端的控制方式, 影响了控制效率.

\section{2 几余发送}

\subsection{1 拷贝冗余}

若网络的播撒密度较大,则节点可以在转发时创建一个数据包的多个搭贝,并同时向多个邻居节点转发, 以 数据冗余的方式来保证传输可靠性. 该方法的核心问题是,节点如何根据当前的网络状况确定选择合适和一定 量的邻居节点来满足可靠性要求.

AFS 协议 ${ }^{[33]}$ 中,初始阶段节点使用洪泛广播数据包.网关收集一段时间的数据之后,按照包的序列号统计计 算每个流的实际可靠率, 并按照实际可靠率和可靠性要求的比率来设定相关节点的转发概率, 再将新的设置通 过控制包发送给节点进行更新.这种基于网关集中控制的方法比较简单,缺点在于需要网关发送大量的控制包 进入网络,加大了网络负载和能耗.同时,统一的调整模式使得单个节点不能根据当前自身的网络状况灵活地调 
整转发概率,在大规模网络中节点等待网关反馈时间较长.

Reinform 协议 ${ }^{[34]}$ 根据传输节点的可靠性要求和节点的丢包率来选择转发路径.对传输节点 $i$, 定义转发路 径数量为

$$
P_{i}=\frac{\log \left(1-r_{i}\right)}{\log \left(1-\left(1-e_{i}\right)^{h_{i}}\right)}-\left(1-e_{i}\right),
$$

其中, $h_{i}$ 为节点 $i$ 到网关的跳数, $e_{i}$ 为节点 $i$ 的丢包率, $r_{i}$ 为可靠性要求. 若 $i$ 是源节点 $S$, 则可以设置 $r_{s}$ 的值, 其他转 发节点通过下面的公式计算:

$$
r_{i}=1-\left(1-\left(1-e_{s}\right)^{h_{s}-1}\right)^{P} .
$$

为了达到路径选择的数量要求, 节点 $i$ 从邻居节点中按照 $1:\left(1-e_{i}\right):\left(1-e_{i}\right)^{2}$ 的比例选择距网关跳数分别为 $\left(h_{i}-1\right), h_{i},\left(h_{i}+1\right)$ 的节点, 其中, 优先选择跳数较少的节点. 转发策略定义为: 从跳数等于 $\left(h_{i}-1\right)$ 的邻居节点中随机选 择一个节点作为缺省下一跳节点参与转发, 其他节点若收到数据包, 则根据自身计算的 $P$ 值大小来决定是否参 与转发, 若 $P \geq 1$ 则转发, 否则按照 $P$ 值的概率决定是否转发数据包. 该协议的缺点在于选择下一跳转发邻居节点 时进行随机选取,而没有考虑到被选节点的能耗、丢包率等因素. 另外, 选取上一跳节点作为转发节点的做法在 某些情况下可能导致路由回路的出现.

MMSPEED 协议 ${ }^{[35]}$ 根据相邻的上下游节点对之间的丢包率来选择转发节点. 对相邻节点 $i$ 和 $j$, 节点 $i$ 统计 $i$ 到 $j$ 的丢包率为 $e_{i j}$, dist 表示节点间距离, 定义数据包从 $i$ 经 $j$ 到目标节点 $d$ 的可达概率为

定义从 $i$ 到 $j$ 的总可达概率为

$$
R P_{i, j}^{d}=\left(1-e_{i, j}\right)\left(1-e_{i, j}\right)^{\left[\text {dist } j, d_{j} / \text { dist }_{i, j}\right]} .
$$

$$
T R P=1-(1-T R P)\left(1-R P_{i, j}^{d}\right) .
$$

定义 $P^{r e q}$ 为可靠概率要求, 初始值由源节点根据包的类型或属性等来设定, $T R P$ 初始值为 0 . 节点 $i$ 从邻居节 点中不断选择可达概率较大的节点作为转发节点, 直到满足 $T R P>P^{r e q}$ 为止. 同时, 节点 $i$ 根据当前自身的 $P^{r e q}$ 值 计算对这些转发节点的可靠概率要求,并捎带在数据包中发送,各转发节点将根据该值重复以上选择过程. 若下 游的某节点在转发选择时发现无法满足 $T R P>P^{\text {req }}$,则通过后压消息的方式反馈给其上游节点,上游节点在之后 的数据传输中将重新调整对各节点的可靠性要求比例,降低对该节点的可靠概率要求.MMSPEED 将全局的可 靠问题转化为节点根据本地网络状态进行的判断,并能够在本地判断失误时提供快速而有效的调整机制,缺点 在于未考虑转发节点选择时的能耗等因素.

GRAB 协议 ${ }^{[366}$ 将基于梯度场的路由和受限广播结合起来,在发送报文时在包头中嵌入如下信息段:源节点 赋于数据包的 credit 值 $\alpha$, 源节点的梯度值 $C_{\text {source, }}$, 从源端传输到当前节点消耗的能量总和 $P_{\text {consumed, }}$, 广播该数据包 的邻居节点的梯度值 $C_{\text {sender }}$. 每个节点在收到邻居节点的数据广播之后, 将比较 $R_{\alpha}$ 和 $R_{\text {thresh }}$, 其中:

$$
\begin{gathered}
R_{\alpha}=\frac{\alpha-\left(P_{\text {consumed }}+C_{\text {receiver }}-C_{\text {source }}\right)}{\alpha}, \\
R_{\text {thresh }}=\left(\frac{C_{\text {receiver }}}{C_{\text {source }}}\right)^{2} .
\end{gathered}
$$

若 $R_{\alpha}>R_{\text {thresh }}$, 则节点控制发送功率将数据转发给离自身最近的 3 个邻居节点; 若 $R_{\alpha}<R_{\text {thresh }}$, 则节点只将数据 包发送给按照梯度路由算法得到的单个最优邻居转发节点. 若一个节点收到了某个数据包的多份楛贝,则只发 送一份掿贝.该协议将广播范围缩小在从数据源到网关的带状区域内,在提高可靠性的同时还考虑了网络的能 耗问题. 其缺点在于没有考虑到单个节点丢包率的不同, 另外,设置转发邻居数量为 3 , 仅适用于丢包率较小的 情况.

\section{2 .2 编码几余}

擦除码 (erasure code) 是一种容错机制, 它可以将 $m$ 个源数据编码为 $n(n>m)$ 个新数据, 使用这 $n$ 个新数据中 任意的 $m$ 个编码数据均可重构原来 $m$ 个源数据. 这种方法作为一种前向纠错(forward error correction)技术主要 
应用在通信网络传输中避免包的丢失,使用比拷贝方式更少的带宽和存储空间,提供与其效果相近的可靠性. 文 献[37,38]分别将它与 BVR 路由 ${ }^{[39]}$ 和 Directed Diffusion 路由 ${ }^{[40]}$ 结合, 以提高 WSN 的传输性能.

文献[37]中给出了使用 Reed-Solomon 码来实现擦除码算法的基本原理:将给定的数据分解为矩阵 $W_{m \times 1}$ 中 的元素,同时,选择 Vandermonde 矩阵 $A_{n \times m}$ 作为编码生成矩阵,也即满足 $A(i, j)=x_{i}^{j-1}$.经过运算可得

$$
P_{n \times 1}=\left(\begin{array}{c}
p\left(x_{1}\right) \\
p\left(x_{2}\right) \\
p\left(x_{3}\right) \\
\vdots \\
\vdots \\
p\left(x_{n-1}\right) \\
p\left(x_{n}\right)
\end{array}\right)=\left(\begin{array}{cccc}
1 & x_{1} & \cdots & x_{1}^{m-1} \\
1 & x_{2} & \cdots & x_{2}^{m-1} \\
1 & x_{3} & \cdots & x_{3}^{m-1} \\
\vdots & \vdots & & \vdots \\
\vdots & \vdots & & \vdots \\
1 & x_{n-1} & \cdots & x_{n-1}^{m-1} \\
1 & x_{n} & \cdots & x_{n}^{m-1}
\end{array}\right)\left(\begin{array}{c}
w_{0} \\
w_{1} \\
\vdots \\
w_{m-1}
\end{array}\right) .
$$

从矩阵 $P$ 中任意选取 $m$ 个元素组成矩阵 $P^{\prime}$, 就可以通过选择对应的编码矩阵的子方矩阵 $A^{\prime}$, 求解出 $W_{m \times 1}=\left(A^{\prime}\right)^{-1} P^{\prime}$.

该方法的缺点在于对传感器节点的计算处理能力要求较高.为了将该方法应用到资源受限的 WSN 中, 文 献[37]提出,采用系统码(矩阵 $A$ 中设置部分元素为 0 , 不进行编码运算),将相同序列的编码词放入同一数据包传 输和设置运算表等方法,降低编、解码的复杂度,提高运算效率.

\section{4 无线传感器网络传输控制协议比较}

以上总结了目前 WSN 中一些典型的传输控制协议和方案的设计方法,其中一些协议能够较好地检测和处 理网络拥塞, 另外一些协议则有针对性地解决了传输丢包带来的可靠性问题. 在表 1 中, 我们从多个方面对上述 各种协议进行了综合对比.其中,A:协议;B:类型;C:数据包传输方向;D:(拥塞/丢包)检测方法;E:(拥塞/丢包)反馈 方法;F:传输开销;G:计算开销;H:是否需要添加特殊硬件支持;I:可靠性度量;J:控制方式.

Table 1 Comparison of transmission control protocols in wireless sensor networks

表 1 WSN 传输控制协议比较

\begin{tabular}{|c|c|c|c|c|c|c|c|c|c|}
\hline $\mathrm{A}$ & $\mathrm{B}$ & $\mathrm{C}$ & $\mathrm{D}$ & $E$ & $\mathrm{~F}$ & $G$ & $\mathrm{H}$ & $\mathrm{I}$ & $\mathrm{J}$ \\
\hline $\mathrm{ESRT}^{[9]}$ & Congestion & Sensor->Sink & Buffer & ICN & Moderate & Low & No & Event-Based & Centralized \\
\hline PORT $^{[10]}$ & Congestion & Sensor- $>$ Sink & & ECN & Moderate & Moderate & No & Event-Based & Both \\
\hline IFRC $^{[18]}$ & Congestion & Sensor- $>$ Sink & Buffer & ICN & Low & Low & No & Event-Based & Distributed \\
\hline Fusion $^{[17]}$ & Congestion & Sensor- $>$ Sink & Buffer & ICN & Low & Low & No & Event-Based & Distributed \\
\hline $\mathrm{CCF}^{[3]}$ & Congestion & Sensor- $>$ Sink & Buffer & ICN & Low & Moderate & No & Event-Based & Distributed \\
\hline Buffer-Based $^{[19]}$ & Congestion & Sensor- $>$ Sink & Buffer & ICN & Low & Low & No & Event-Based & Distributed \\
\hline $\mathrm{PCCP}^{[16]}$ & Congestion & Sensor- $>$ Sink & Congestion degree & ICN & Moderate & Moderate & No & Event-Based & Distributed \\
\hline $\operatorname{CODA}^{[15]}$ & Congestion & Sensor- $>$ Sink & Buffer \& Channel & ECN & High & Moderate & No & Event-Based & Both \\
\hline $\mathrm{ARC}^{[20]}$ & Congestion & Sensor- $>$ Sink & Buffer & ICN & Moderate & Low & No & Packet-Based & Distributed \\
\hline $\mathrm{CAR}^{[21]}$ & Congestion & Sensor- $>$ Sink & & & Moderate & Low & No & Packet-Based & Both \\
\hline $\mathrm{BGR}^{[22]}$ & Congestion & Sensor- $>$ Sink & Buffer \& Channel & ICN & Low & Low & No & Packet-Based & Distributed \\
\hline CONCERT $^{[24]}$ & Congestion & Sensor- $>$ Sink & & & Low & High & No & Event-Based & Distributed \\
\hline PREI $^{[25]}$ & Congestion & Sensor- $>$ Sink & & & Low & High & No & Event-Based & Distributed \\
\hline Siphon ${ }^{[26]}$ & Congestion & Sensor- $>$ Sink & Buffer \& Channel & ECN & Low & Low & Yes & Packet-Based & Centralized \\
\hline $\mathrm{PSFQ}^{[28]}$ & Reliability & Sink->Sensor & Packet id & NACK & Moderate & Low & No & Packet-Based & Distributed \\
\hline GARUDA $^{[30]}$ & Reliability & Sink->Sensor & Packet id & NACK & High & Low & Yes & Packet-Based & Distributed \\
\hline $\operatorname{RMST}^{[29]}$ & Reliability & Sensor- $>$ Sink & Packet id & NACK & Low & Low & No & Packet-Based & Distributed \\
\hline $\mathrm{RBC}^{[31]}$ & Reliability & Sensor- $>$ Sink & Buffer id & IACK \& ACK & Low & Moderate & No & Packet-Based & Distributed \\
\hline BRTM $^{[32]}$ & Reliability & Bidirectional & Packet id & NACK \& ACK & Low & Low & No & Both & Centralized \\
\hline $\mathrm{AFS}^{[33]}$ & Reliability & Sensor->Sink & & & High & Low & No & Packet-Based & Centralized \\
\hline Reinform $^{[34]}$ & Reliability & Sensor- $>$ Sink & & & High & Moderate & No & Packet-Based & Distributed \\
\hline MMSPEED $^{[35]}$ & Reliability & Sensor- $>$ Sink & & & High & Moderate & No & Packet-Based & Distributed \\
\hline GRAB $^{[36]}$ & Reliability & Sensor- $>$ Sink & & & Moderate & Moderate & No & Packet-Based & Distributed \\
\hline ErasureCode $^{[37]}$ & Reliability & Sensor- $>$ Sink & & & High & High & No & Packet-Based & Distributed \\
\hline
\end{tabular}

通过表 1 的比较,我们可以从整体上对 WSN 的传输控制协议有一个比较清晰而完整的认识,有助于我们更 加全面、深入地了解和吸收已有的研究成果,并在此基础上思考和解决相关问题. 


\section{5 总结与展望}

本文综合国内外的相关研究,从拥塞控制和可靠保证两个方面系统地阐述了当前典型的 WSN 传输控制协 议及其特点. 通过对各种协议的比较和分析, 我们认为 WSN 的传输控制协议在以下几个方面还有待进一步深入 研究:

1) 设计跨层协作的传输控制协议. 在 WSN 中传输控制任务不能仅仅依靠传输层来完成, 传感器节点协议 栈中的各个层次应在传输层控制机制的协调下进行充分的交互与协作, 共同支持和保证数据的可靠传输.

2) 提高传输控制协议的综合控制能力.在 WSN 应用过程中,网络中拥塞和丢包的现象可能会同时发生并 互相影响,网关和节点之间也会频繁地进行数据交互. 目前的控制协议大多只对单个数据流方向上的一种问题 (拥塞或丢包)进行处理,新的传输控制协议应提供全面的综合控制机制.

3) 基于多优先级的控制策略.网络中出现不同的数据流对可靠性的要求可能会不同 ${ }^{[16,33]}$, 需要在协议设计 时考虑对优先级问题的处理.

4) 传输控制协议需要提供公平性保证.在确保传输效率的同时,网络中的多个数据流应按照事先定义的公 平性原则分享无线信道进行数据传输 ${ }^{[3,18]}$.

5) 支持 WSN 中以数据为中心的设计方案. WSN 以数据为中心的特点带来了一些新的设计方案, 如无独立 地址的节点设计 ${ }^{[1,12]}$ 、数据的在网处理和分簇网络结构 ${ }^{[11]}$ 等, 新的传输控制协议需要提供对这些方案的支持.

6) 提供对节点移动性的支持. 目前,WSN 传输控制协议基本都假定传感器节点和网络是静态的.但在战场、 物流等应用中, 节点的移动会给网络传输带来更多不可靠因素, 加重丢包现象的发生, 需要设计具有更高处理效 率和更快处理速度的控制协议。

\section{References:}

[1] Ren FY, Huang HN, Lin C. Wireless sensor networks. Journal of Software, 2003,14(7):1282-1290 (in Chinese with English abstract). http://www.jos.org.cn/1000-9825/14/1282.htm

[2] Wang CG, Sohraby K, Li B, Hu YM. A survey of transport protocols for wireless sensor networks. IEEE Network Magazine, 2006,20(3): 34-40.

[3] Ee CT, Bajcsy R. Congestion control and fairness for many-to-one routing in sensor networks. In: Stankovic AJ, Arora A, Govindan R, eds. Proc. of the 2nd ACM Conf. on Embedded Networked Sensor Systems (SenSys). Baltimore: ACM Press, 2004. $148-161$.

[4] Feng YJ, Sun LM, Qian HL, Song C. Improving TCP performance over MANET: A survey. Journal of Software, 2005,16(3): 434-444 (in Chinese with English abstract). http://www.jos.org.cn/1000-9825/16/434.htm

[5] Zhang M, Wu JP, Lin C. Survey on Internet end-to-end congestion control. Journal of Software, 2002,13(3):354-363 (in Chinese with English abstract). http://www.jos.org.cn/1000-9825/13/354.pdf

[6] Stevens W. TCP slow start, congestion avoidance, fast retransmit, and fast recovery. RFC 2001, 2001. http://rfc.net/rfc2001.html

[7] Karl H, Willig A. Protocols and Architectures for Wireless Sensor Networks. England: John Wiley \& Sons, 2005.

[8] Li L, Zhou XS, Li SN, Li ZG, Zheng Y. Study and comparison of congestion control algorithms in wireless sensor network. Application Research of Computers, 2006,23(3):11-13 (in Chinese with English abstract).

[9] Sankarasubramaniam Y, Akan OB, Akyildiz I. ESRT: Event-to-Sink reliable transport in wireless sensor networks. In: Gerla M, Ephreides A, Srivastava M, eds. Proc. of the 4th ACM Symp. on Mobile Ad Hoc Networking and Computing (MobiHoc). Annapolis: ACM Press, 2003. 177-188.

[10] Zhou YF, Lyu MR. PORT: A price-oriented reliable transport protocol for wireless sensor networks. In: Binger VR, Tsai JJ, Wong EW, eds. Proc. of the 16th IEEE Int'l Symp. on Software Reliability Engineering (ISSRE). Chicago: IEEE Press, 2005. 117-126.

[11] Fang WW, Liu Y, Wu J, Qian DP. An on-demand address allocation scheme for query based sensor networks. In: Kim K, Kakuda Y, Moeller E, eds. Proc. of the 8th Int'l Symp. on Autonomous Decentralized Systems (ISADS). Sedona: IEEE Press, 2007. $173-179$.

[12] Fang WW, Liu Y, Qian DP. EDDS: An efficient data delivery scheme for address-free wireless sensor networks. In: Dini P, Rousseaux F, eds. Proc. of the 6th Int'l Conf. on Networking (ICN). Martinique: IEEE Press, 2007. 7-12. 
[13] Iyer Y, Gandham S, Venkatesan S. STCP: A generic transport layer protocol for wireless sensor networks. In: Dasilva L, Yang YY, Thuel RS, eds. Proc. of the 14th Int'l Conf. on Computer Communications and Networks (ICCCN). San Diego: IEEE Press, 2005. 449-454.

[14] Braun T, Voigt T, Dunkels A. Energy-Efficient TCP operation in wireless sensor networks. PIK Journal, 2005,28(2):93-100.

[15] Wan C, Eisenman S, and Campbell A. CODA: congestion detection and avoidance in sensor networks. In: Akyildiz I, Estrin D, eds. Proc. of the 1st Int'l Conf. on Embedded Networked Sensor Systems. Los Angeles: ACM Press, 2003. 266-279.

[16] Wang CG, Sohraby K, Lawrence V, Li B, Hu YM. Priority-Based congestion control in wireless sensor networks. In: Iyengar SS, Ni ML, Tsai JJ, eds. Proc. of the IEEE Int'l Conf. on Sensor Networks, Ubiquitous, and Trustworthy Computing (SUTC). Taichung: IEEE Press, 2006. 22-31.

[17] Hull B, Jamieson K, Balakrishnan H. Mitigating congestion in wireless sensor networks. In: Stankovic AJ, Arora A, Govindan R, eds. Proc. of the 2nd ACM Conf. on Embedded Networked Sensor Systems (SenSys). Baltimore: ACM Press, 2004. 134-147.

[18] Rangwala S, Gummadi R, GummadiR, Govindan R, Psounis K. Interference-aware fair rate control in wireless sensor networks. In: Rizzo L, Anderson ET, eds. Proc. of the ACM SIGCOMM Symp. on Network Architectures and Protocols. Pisa: ACM Press, 2006. 63-74.

[19] Chen SG, Yang N. Congestion avoidance based on lightweight buffer management in sensor networks. IEEE Trans. on Parallel and Distributed Systems, 2006,17(9):934-946.

[20] Kang J, Zhang YY, Nath B, Yu SC. Adaptive resource control scheme to alleviate congestion in sensor networks. In: Heinzelman W, Krishnamachari B, eds. Proc. of the 1st Workshop on Broadband Advanced Sensor Networks (BASENETS). San Jose: IEEE Press, 2004.

[21] Kumar R, Rowaihy H, Cao GH, Anjum F, Yener A, La Porta T. Congestion aware routing in sensor networks. Technical Report, 2006. http://nsrc.cse.psu. edu/tech_report/NAS-TR-0036-2006.pdf

[22] Popa L, Raiciu C, Stoica.I, Rosenblum DS. Reducing congestion effects in wireless networks by multipath routing. In: Almeroth CK, Higashino T, eds. Proc. of the 14th IEEE Int'l Conf. on Network Protocols (ICNP). Santa Barbara: IEEE Press, 2006. 96-105.

[23] Sun LM, Li JZ, Chen Y, Zhu HS. Wireless Sensor Networks. Beijing: Tsinghua University Press, 2005 (in Chinese).

[24] Gulluccio L, Campbell AT, Palozzo S. CONCERT: Aggregation-Based congestion control for sensor networks. In: Redi J, Balakrishnan H, Zhao F, eds. Proc. of the 3rd ACM Conf. on Embedded Networked Sensor Systems (SenSys). San Diego: ACM Press, 2005. 274-275.

[25] Ngai E, Zhou YF, Lyu MR, Liu JC. Reliable reporting of delay-sensitive events in wireless sensor-actuator networks. In: Perkins EC, Wu J, eds. Proc. of the 3rd IEEE Int'l Conf. on Mobile Ad-hoc and Sensor Systems (MASS). Vancouver: IEEE Press, 2006. $101-108$.

[26] Wan YC, Eisenman SB, Campbell AT, Crowcroft J. Siphon: Overload traffic management using multi-radio virtual sinks in sensor networks. In: Redi J, Balakrishnan H, Zhao F, eds. Proc. of the 3rd ACM Conference on Embedded Networked Sensor Systems (SenSys). San Diego: ACM Press, 2005. 116-129.

[27] Crossbow wireless sensor networks: Products overview. http://www.xbow.com.cn/wsn/product/default.asp

[28] Wan CY, Campbell A, Krishnamurthy L. PSFQ: A reliable transport protocol for wireless sensor networks. In: Raghavendra SC, Silvalingam MK, eds. Proc. of ACM Int'l Workshop on Wireless Sensor Networks and Applications. Atlanta: ACM Press, 2002. $1-11$.

[29] Stann F, Heidemann. RMST: Reliable data transport in sensor networks. In: Proc. of the 1st Int'l Workshop on Sensor Net Portocols and Applications (SNPA). Anchorage: IEEE Press, 2003. 102-112.

[30] Park SJ, Vedantham R, Sivakumar R and Akyildiz I. A scalable approach for reliable downstream data delivery in wireless sensor networks. In: Murai J, Perkins C, Tassiulas L, eds. Proc. of the 5th ACM Symp. on Mobile Ad Hoc Networking and Computing (MobiHoc). Tokyo: ACM Press, 2004. 78-89.

[31] Zhang HW, Arora A, Choi YR, Gouda MG. Reliable bursty convergecast in wireless sensor networks. In: Kumar RP, ed. Proc. of the 6th ACM Symp. on Mobile Ad Hoc Networking and Computing (MobiHoc). Urbana-Champaign: ACM Press, 2005. 266-276. 
[32] Tezcan N, Wang WY, Chow MY. A bidirectional reliable transport mechanism for wireless sensor networks. In: Porta LT, Lu SW, eds. Proc. of the IEEE Military Communications Conf. (MILCOM). Atlantic: IEEE Press, 2005. 1193-1199.

[33] Bhatnagar S, Deb B, Nath B. Service differentiation in sensor networks. In: Proc. of the 4th Int'l Symp. on Wireless Personal Multimedia Communications. Aalborg: IEEE Press, 2001.

[34] Deb B, Bhatnagar S, Nath B. Reinform: reliable information forwarding using multiple paths in sensor networks. In: Proc. of the 28th IEEE Int'l Conf. on Local Computer Networks (LCN). Bonn: IEEE Press, 2003. 406-415.

[35] Felemban E, Lee CG, Ekici E, Boder R, Vural S. Probabilistic QoS guarantee in reliability and timeliness domains in wireless sensor networks. In: Znati T, Knightly E, Makki K, eds. Proc. of 24th Annual Joint Conf. of the IEEE Computer and Communications Societies (INFOCOM). Miami: IEEE Press, 2005. 2646-2657.

[36] Ye F, Zhong G, Lu SW, Zhang LX. Gradient broadcast: A robust data delivery protocol for large scale sensor networks. ACM Wireless Networks, 2005,11(3):285-298.

[37] Kim.S, Fonseca R, Culler D. Reliable transfer on wireless sensor networks. In: Znati T, Lee SJ, Mohapatra P, Sivalingam K, eds. Proc. of the 1st IEEE Int'l Conf. on Sensor and Ad Hoc Communications and Networks (SECON). Santa Clara: IEEE Press, 2004. 449-459.

[38] Djukic P, Valaee S. Reliable and energy efficient transport layer for sensor networks. In: Kero T, Gupta KP, Figueira N, eds. Proc. of the Global Telecommunications Conf. (GLOBECOM). San Francisco: IEEE Press, 2006. 1-5.

[39] Fonseca R, Ratnasamy S, Culler D, Shenker S, Stoica I. Beacon vector routing: Scalable point-to-point in wireless sensornets. Technical Report, IRB-TR-04-012, Berkeley: Intel Research, 2004.1-14.

[40] Intanagonwiwat C, Govindan R, Estrin D, Heidemann J. Directed diffusion for wireless sensor networking. IEEE/ACM Trans. on Networking, 2003,11(1):2-16.

[41] Shen B, Zhang SY, Zhong YP. Cluster-Based routing protocols for wireless sensor networks. Journal of Software, 2006,17(7):1588-1600 (in Chinese with English abstract). http://www.jos.org.cn/1000-9825/17/1588.htm

\section{附中文参考文献:}

[1] 任丰原,黄海宁,林闯.无线传感器网络.软件学报, 2003,14(7):1282-1290. http://www.jos.org.cn/1000-9825/14/1282.htm

[4] 冯彦君,孙利民,钱华林,宋成.MANET 中 TCP 改进研究综述.软件学报,2005,16(3):434-444. http://www.jos.org.cn/1000-9825/ 16/434.htm

[5] 章录, 吴建平,林闯.互联网端到端拥塞控制研究综述.软件学报,2002,13(3):354-363. http://www.jos.org.cn/1000-9825/13/ 354.pdf

[8] 李凌,周兴社,李士宁,李志刚,郑勇.基于无线传感器网络的拥塞控制算法的研究与比较.计算机应用研究,2006,23(3):11-13.

[23] 孙利民,李建中,陈渝,朱红松.无线传感器网络.北京:清华大学出版社,2005.

[41] 沈波,张世永, 钟亦平.无线传感器网络分簇路由协议.软件学报,2006,17(7):1588-1600. http://www.jos.org.cn/1000-9825/17/ 1588.htm

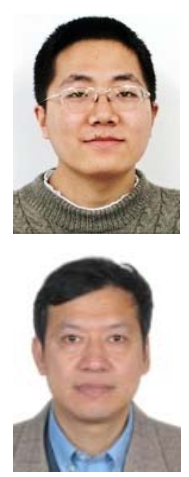

方维维 $(1981-)$, 男,安徽芜湖人,博士生, 主要研究领域为无线传感器网络.

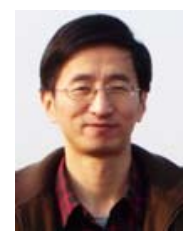

刘轶(1968-),男,博士,副教授,主要研究 领域为无线传感器网络,嵌入式系统.

钱德沛(1952-),男,教授,博士生导师,CCF 高级会员,主要研究领域为高性能计算机, 网络技术,计算网络管理,性能测量,无线 传感器网络。 